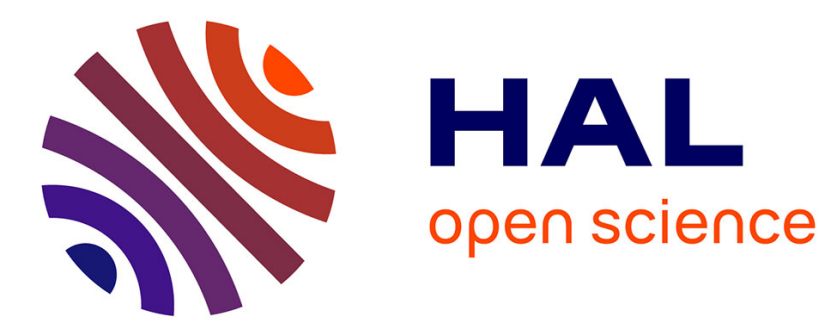

\title{
Feedback linearization for the DC voltage control of a VSC-HVDC terminal
}

Yijing Chen, Gilney Damm, Abdelkrim Benchaib, Françoise

Lamnabhi-Lagarrigue

\section{- To cite this version:}

Yijing Chen, Gilney Damm, Abdelkrim Benchaib, Françoise Lamnabhi-Lagarrigue. Feedback linearization for the DC voltage control of a VSC-HVDC terminal. ECC 2014 - European Control Conference, Jun 2014, Strasbourg, France. pp.1999-2004, 10.1109/ECC.2014.6862439 . hal-01090320

\section{HAL Id: hal-01090320 \\ https://hal.science/hal-01090320}

Submitted on 18 Sep 2020

HAL is a multi-disciplinary open access archive for the deposit and dissemination of scientific research documents, whether they are published or not. The documents may come from teaching and research institutions in France or abroad, or from public or private research centers.
L'archive ouverte pluridisciplinaire HAL, est destinée au dépôt et à la diffusion de documents scientifiques de niveau recherche, publiés ou non, émanant des établissements d'enseignement et de recherche français ou étrangers, des laboratoires publics ou privés. 


\title{
Feedback Linearization for the DC Voltage Control of a VSC-HVDC Terminal
}

\author{
Yijing Chen ${ }^{1}$, Gilney Damm ${ }^{2}$, Abdelkrim Benchaib ${ }^{3}$, Françoise Lamnabhi-Lagarrigue ${ }^{1}$
}

\begin{abstract}
This paper investigates the use of feedback linearization to develop a control system for regulating the DC voltage and the reactive power of a VSC terminal. Firstly, a direct-quadrature $(d q)$ state-space model of the VSC terminal is established. By analyzing the zero dynamics of the system, the static feedback linearization can be applied when the VSC terminal operates in inversion mode (power flowing from the DC side to the AC one). However, the system is not feedback linearizable when the VSC works in rectification mode (power flowing from the $\mathrm{AC}$ side to the $\mathrm{DC}$ one). Then, based on a simplified model, a new dynamic is introduced by an additional control variable which yields a higher order system, allowing the terminal to operate in rectification mode. Numerical simulations are carried out to verify the validity of the proposed control system.
\end{abstract}

\section{INTRODUCTION}

In the past decades, significant advances have been made in the development of high power devices for Voltage Source Converter based High Voltage Direct Current (VSC-HVDC) systems. These systems are capital to attain the objective of integrating large-scale offshore renewable energy sources. One of the most significant progress was the improvement of high rated transistors, such as insulated gate bipolar transistors (IGBT), that made VSC-HVDC systems operate in a flexible, efficient and reliable way. With these types of semiconductor devices, a second degree of freedom is given since they can be turned both on and off. This additional controllability gives many advantages. It is possible to control the reactive power independently of the active power and power transmission can be bidirectional. The application of Pulse Width Modulation (PWM) [1] improves the harmonic distortion of the converter with less low frequency harmonics and gives a faster response. VSC-HVDC systems have also the potential benefits of transferring power flows to/from a weak AC network such as wind farms and solar plants while improving the stability and the robustness of the overall system [2], [3], [4], [5].

Many studies have been devoted to the control design of VSC-HVDC systems. Traditional control structure involves the field-oriented vector control technique based on several PI controllers [6]. Generally speaking, two PI controllers

This research is supported by the French national project WINPOWER (ANR-10-SEGI-016).

${ }^{1}$ Y. Chen and F. Lamnabhi-Lagarrigue are with Laboratoire des Signaux et Systèmes (LSS), Supélec, 3 rue Joliot-Curie, 91192 Gif-surYvette, France (tel: +33 1698517 77, e-mail: yijing.chen@1ss.supelec.fr, francoise.lamnabhi-lagarrigue@lss.supelec.fr).

${ }^{2} \mathrm{G}$. Damm is with Laboratoire IBISC, Université d'Evry-Val d'Essonne, 40 rue du Pelvoux, 91020 Evry, France (e-mail: gilney.damm@ibisc.fr).

${ }^{3}$ A. Benchaib is with ALSTOM GRID/CNAM, France (e-mail: abdelkrim.benchaib@alstom.com). are used to control the $d q$ currents. According to different control objectives, an additional PI controller is dedicated to controlling the DC voltage or the active power. However, there exists a limitation in this conventional control method [7]. Moreover, when the dynamics of the currents and the DC voltage are close to each other, such kind of vector control is not so efficient [8]. Other possibilities have been recently studied. For example, a passivity property of the dynamical system has been exploited in [9]. Though the global stability of the system is ensured, the control strategy in [9] is based on the equilibrium values of the system which need to be calculated in advance and are strongly dependent on parameters and measurements.

In this work, we propose a new control system based on feedback linearization theory, which ensures that the converter can operate well in both rectification mode and inversion mode. The zero dynamics of the system are derived and analyzed in this paper, which is very important to help us understanding the behavior of the system.

This paper is organized as follows. A synchronous $d q$ reference frame based state-space model is introduced in Section II. The control structure is built in Section III where the zero dynamics of the system are analyzed. It is found that the system has the property of being feedback linearizable in inversion mode but not in rectification mode. Then, a control law based on a simplified model is derived for the converter in rectification mode. Simulation results are presented in Section IV. Finally, conclusions are drawn in Section V.

\section{MOdELING OF A VSC TERMinAL}

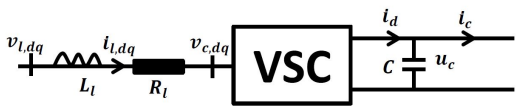

Fig. 1. A simplified configuration of a VSC terminal.

A simplified representation of a VSC terminal is shown in Fig. 1 where a small resistance $R_{l}$ and a large inductance $L_{l}$ represent the phase reactor which dominates the dynamics of the converter on the $\mathrm{AC}$ side and $C$ is the $\mathrm{DC}$ capacitor whose size determines the dynamics on the DC side. $v_{l, d q}$ are the voltages of the AC network, $v_{c, d q}$ are the voltages on the AC side of the converter, $i_{l, d q}$ are the currents through the phase reactor, $u_{c}$ is the DC voltage across the DC capacitor and $i_{c}$ is the measurable DC current representing the active power demand or supply from the connected DC grid. By convention, $i_{c}$ is positive if the power flows from the $\mathrm{AC}$ side to the DC side. 
A state-space model of the VSC terminal established in a synchronous $d q$ frame is given by [10]:

$$
\begin{aligned}
\frac{d i_{l d}}{d t} & =-\frac{R_{l}}{L_{l}} i_{l d}+\omega i_{l q}-\frac{1}{2 L_{l}} M_{d} u_{c}+\frac{v_{l d}}{L_{l}} \\
\frac{d i_{l q}}{d t} & =-\frac{R_{l}}{L_{l}} i_{l q}-\omega i_{l d}-\frac{1}{2 L_{l}} M_{q} u_{c}+\frac{v_{l q}}{L_{l}} \\
\frac{d u_{c}}{d t} & =-\frac{1}{C} i_{c}+\frac{1}{C} \frac{3}{4}\left(M_{d} i_{l d}+M_{q} i_{l q}\right)
\end{aligned}
$$

where $\omega$ is the angular frequency of the AC network. Pulsewidth modulation (PWM) technology is applied to generate the converter voltages $v_{c, d q}$ where $M_{d q}$ are the modulation indices as the control inputs. $v_{c, d q}$ are controlled by $M_{d q}$ :

$$
v_{c, d q}=\frac{1}{2} M_{d q} u_{c}
$$

The active and reactive power on the AC side of the converter are expressed as:

$$
\begin{aligned}
P_{l} & =\frac{3}{2} v_{l d} i_{l d} \\
Q_{l} & =-\frac{3}{2} v_{l d} i_{l q}
\end{aligned}
$$

since the chosen $d q$ reference frame makes the d-axis fixed to the $\mathrm{AC}$ area voltage, i.e. $v_{l d}=V_{l, \mathrm{rms}}$ and $v_{l q}=0$.

\section{CONTROL STRATEGY}

As presented in the state-space model (1)-(3), there are two degrees of freedom. Thus, at least two variables can be controlled. In this paper, $u_{c}$ and $Q_{l}$ are chosen to be regulated at their desired values $u_{c}^{*}$ and $Q_{l}^{*}$. As seen in (6), controlling $Q_{l}$ is equivalent to regulating $i_{l q}$ at $i_{l q}^{*}$ which satisfies

$$
i_{l q}^{*}=-\frac{2 Q_{l}^{*}}{3 v_{l d}}
$$

In this paper, the control objective is to make $u_{c}$ and $i_{l q}$ track their references $u_{c}^{*}$ and $i_{l q}^{*}$, respectively. An important feature of the VSC terminal is that the system can operate at unity power factor. For simplicity, $i_{l q}^{*}$ is set to zero.

In this paper, according to the direction of the power flows, two control laws are developed based on feedback linearization theory.

\section{A. Static feedback linearization control}

By defining $\left(\begin{array}{lll}x_{1} & x_{2} & x_{3}\end{array}\right) \triangleq\left(\begin{array}{lll}i_{l d} & i_{l q} & u_{c}\end{array}\right)$, we rewrite the system (1)-(3) as:

$$
\dot{x}=f(x)+g_{d} M_{d}+g_{q} M_{q}
$$

where $f(x), g_{d}$ and $g_{q} \in \mathbb{R}^{3}$ are easily found.

According to the control objective, we first define the outputs as $y_{1}=\left(\begin{array}{ll}u_{c} & i_{l q}\end{array}\right)^{T}$. Consider the square system (8) where the number of control inputs is equal to the number of outputs. It is easy to find that the relative degree of each output is $r=1$. Let $J$ be an $2 \times 2$ matrix such that:

$$
J=\left(\begin{array}{cc}
L_{g_{d}}\left(i_{l q}\right) & L_{g_{q}}\left(i_{l q}\right) \\
L_{g_{d}}\left(u_{c}\right) & L_{g_{q}}\left(u_{c}\right)
\end{array}\right)
$$

where the Lie derivatives of each output with respect to $g_{d}$, $g_{q}$ and $f$ are:

$$
\begin{aligned}
L_{g_{d}}\left(i_{l q}\right) & =0 \\
L_{g_{d}}\left(u_{c}\right) & =\frac{3 i_{l d}}{4 C} \\
L_{g_{q}}\left(i_{l q}\right) & =-\frac{u_{c}}{2 L_{l}} \\
L_{g_{q}}\left(u_{c}\right) & =\frac{3 i_{l q}}{4 C}
\end{aligned}
$$

$J$ is non-singular under the condition $u_{c} i_{l d} \neq 0$. Therefore, a nonlinear feedback control can be developed as:

$$
\left(\begin{array}{c}
M_{d} \\
M_{q}
\end{array}\right)=J^{-1}\left(\begin{array}{c}
v_{q}-\left(-\frac{R_{l}}{L_{l}} i_{l q}-\omega i_{l d}+\frac{v_{l q}}{L_{l}}\right) \\
v_{u}-\left(-\frac{i_{c}}{C}\right)
\end{array}\right)
$$

with

$$
J^{-1}=\left(\begin{array}{cc}
\frac{2 L_{l}}{u_{c}} \frac{i_{l q}}{i_{l_{d}}} & \frac{4 C}{3 i_{l d}} \\
-\frac{2 L_{l}}{u_{c}} & 0
\end{array}\right)
$$

where $v_{q}$ and $v_{u}$ are the additional control inputs yet to be designed, for instance by using linear technique. The complete system with the controller (10) can be written as:

$$
\begin{aligned}
\frac{d i_{l d}}{d t}= & -\frac{R_{l}}{L_{l}} i_{l d}+\omega i_{l q}+\frac{v_{l d}}{L_{l}} \\
& -\frac{i_{l q}}{i_{l d}}\left(v_{q}-\left(-\frac{R_{l}}{L_{l}} i_{l q}-\omega i_{l d}+\frac{v_{l q}}{L_{l}}\right)\right) \\
& -\frac{2 u_{c} C}{3 i_{l d} L_{l}}\left(v_{u}+\frac{i_{c}}{C}\right) \\
\frac{d i_{l q}}{d t}= & v_{q} \\
\frac{d u_{c}}{d t}= & v_{u}
\end{aligned}
$$

We see that the additional inputs $v_{q}$ and $v_{u}$ are linear with respect to the outputs $i_{l q}$ and $u_{c}$ and hence a linear subspace of dimension two is generated. These new inputs can also transform the system (1)-(3) into a much simpler structure. In addition, (12) represents the internal dynamics of the system. Since $i_{l q}^{*}$ and $u_{c}^{*}$ are the desired trajectories which are given by a higher control level, we design the additional inputs $v_{q}$ and $v_{u}$ as:

$$
\begin{aligned}
\dot{\phi}_{q} & =i_{l q}^{*}-i_{l q} \\
\dot{\phi}_{u} & =u_{c}^{*}-u_{c} \\
v_{q} & =\frac{d i_{l q}^{*}}{d t}+k_{p q}\left(i_{l q}^{*}-i_{l q}\right)+k_{i q} \phi_{q} \\
v_{u} & =\frac{d u_{c}^{*}}{d t}+k_{p u}\left(u_{c}^{*}-u_{c}\right)+k_{i u} \phi_{u}
\end{aligned}
$$

where $k_{p, q u}$ and $k_{i, q u}$ are positive. 
With (17) and (18), the closed-loop system is written as:

$$
\begin{aligned}
\dot{\phi}_{q} & =i_{l q}^{*}-i_{l q} \\
\dot{\phi}_{u} & =u_{c}^{*}-u_{c} \\
\frac{d i_{l d}}{d t}= & -\frac{R_{l}}{L_{l}} i_{l d}+\omega i_{l q}+\frac{v_{l d}}{L_{l}} \\
& -\frac{i_{l q}}{i_{l d}}\left(\frac{d i_{l q}^{*}}{d t}+k_{p q}\left(i_{l q}^{*}-i_{l q}\right)+k_{i q} \phi_{q}\right. \\
& \left.-\left(-\frac{R_{l}}{L_{l}} i_{l q}-\omega i_{l d}+\frac{v_{l q}}{L_{l}}\right)\right)-\frac{2 u_{c} C}{3 i_{l d} L_{l}} \times \\
& \left(\frac{d u_{c}^{*}}{d t}+k_{p u}\left(u_{c}^{*}-u_{c}\right)+k_{i u} \phi_{u}+\frac{i_{c}}{C}\right) \\
\frac{d i_{l q}}{d t}= & \frac{d i_{l q}^{*}}{d t}+k_{p q}\left(i_{l q}^{*}-i_{l q}\right)+k_{i q} \phi_{q} \\
\frac{d u_{c}}{d t}= & \frac{d u_{c}^{*}}{d t}+k_{p u}\left(u_{c}^{*}-u_{c}\right)+k_{i u} \phi_{u}
\end{aligned}
$$

As seen in (22) and (23), the surfaces $i_{l q}^{*}-i_{l q}=0$ and $u_{c}^{*}-u_{c}=0$ define two manifolds for the system, which are globally attractive. When the outputs of the system are identically equal to their reference values, the behavior of the system is governed by:

$$
\frac{d i_{l d}}{d t}=\frac{1}{L_{l} i_{l d}}\left[v_{l d} i_{l d}-R_{l} i_{l d}^{2}+\frac{1}{i_{l d}}\left(-\frac{2}{3} u_{c}^{*} i_{c}\right)\right]
$$

which is the zero dynamic relative to the outputs $y_{1}$. Linearizing (24) around the equilibrium point $\bar{i}_{l d}$, we obtain

$$
A=\frac{1}{L_{l}} \frac{\frac{2}{3} u_{c}^{*} i_{c}-R_{l}\left(\bar{i}_{l d}\right)^{2}}{\left(\bar{i}_{l d}\right)^{2}}
$$

If $i_{c}<0$, the converter operates in inversion mode and we have a negative $A$, which means that the zero dynamic is locally asymptotically stable. In this case, the system (1)(3) can be locally stabilized under the proposed nonlinear feedback control law [11]. In fact, from (24), there are two equilibrium points of which one is negative and the other is positive. By taking into account technology limitations, only the negative one can be attained. In addition, it is also found that the attraction region of this negative equilibrium point is very large.

If $i_{c}>0$, the converter works as a rectifier. The term $R_{l}\left(\bar{i}_{l d}\right)^{2}$ represents the losses when the power flows through the phase reactor. In practice, the power injected into the HVDC grid represented by $\frac{2}{3} u_{c}^{*} i_{c}$ should be much larger than the losses represented by $R_{l}\left(\bar{i}_{l d}\right)^{2}$. Thus, the term $\frac{2}{3} u_{c}^{*} i_{c}-$ $R_{l}\left(\bar{i}_{l d}\right)^{2}$ should always be positive, which leads to a positive $A$ such that the zero dynamic (24) is unstable. Therefore, we can conclude that the system (1)-(3) is not feedback linearizable in rectification mode. In the next section, we develop a new control law based on a simplified model and feedback linearization technique such that the stability of the converter in rectification mode can be ensured.

\section{B. Feedback linearization control based on the simplified model}

As mentioned in Section III-A, a positive $i_{c}$ results in unstable internal dynamics. One possible approach to tackling this problem is to redefine the outputs as $y_{2}=\left(\begin{array}{ll}i_{l d} & i_{l q}\end{array}\right)^{T}$.
According to (12), $v_{u}$ can be expressed as:

$$
\begin{aligned}
v_{u}=-\frac{i_{c}}{C} & +\frac{3 L_{l}}{2 C u_{c}}\left\{\left[v_{d}-\left(-\frac{R_{l}}{L_{l}} i_{l d}+\omega i_{l q}+\frac{v_{l d}}{L_{l}}\right)\right] i_{l d}\right. \\
& \left.+\left[v_{q}-\left(-\frac{R_{l}}{L_{l}} i_{l q}-\omega i_{l d}+\frac{v_{l q}}{L_{l}}\right)\right] i_{l q}\right\}
\end{aligned}
$$

where $v_{d}$ is an additional control input yet to be determined. With this expression of $v_{u}$, the system (12)-(14) becomes:

$$
\begin{aligned}
\frac{d i_{l d}}{d t}= & v_{d} \\
\frac{d i_{l q}}{d t}= & v_{q} \\
\frac{d u_{c}}{d t}= & -\frac{i_{c}}{C}+\frac{3 L_{l}}{2 C u_{c}}\left\{\left[v_{d}-\left(-\frac{R_{l}}{L_{l}} i_{l d}+\omega i_{l q}+\frac{v_{l d}}{L_{l}}\right)\right] i_{l d}\right. \\
& \left.+\left[v_{q}-\left(-\frac{R_{l}}{L_{l}} i_{l q}-\omega i_{l d}+\frac{v_{l q}}{L_{l}}\right)\right] i_{l q}\right\}
\end{aligned}
$$

We find that $i_{l, d q}$ are linearized and decoupled from the rest of the system and (29) describes the internal dynamics of the system relative to the outputs $y_{2}$. Now the actual control inputs $M_{d q}$ are designed as a nonlinear state feedback control by combining (10) and (26):

$$
\left(\begin{array}{l}
M_{d} \\
M_{q}
\end{array}\right)=\frac{2 L_{l}}{u_{c}}\left(\begin{array}{l}
\left(-R_{l} i_{l d}+\omega L_{l} i_{l q}+v_{l d}\right)-v_{d} \\
\left(-R_{l} i_{l q}-\omega L_{l} i_{l d}+v_{l q}\right)-v_{q}
\end{array}\right)
$$

If $i_{l d}^{*}$ represents a reference signal for $i_{l d}$, we may choose the additional input $v_{d}$ as:

$$
v_{d}=\frac{d i_{l d}^{*}}{d t}+k_{p d}\left(i_{l d}^{*}-i_{l d}\right)+k_{i d} \phi_{d}
$$

where $k_{p i, d}$ are positive and $\phi_{d}$ satisfies:

$$
\dot{\phi}_{d}=i_{l d}^{*}-i_{l d}
$$

With (17) and (31), the closed-loop system is deduced as:

$$
\begin{aligned}
\dot{\phi}_{d}= & i_{l d}^{*}-i_{l d} \\
\frac{d i_{l d}}{d t}= & \frac{d i_{l d}^{*}}{d t}+k_{p d}\left(i_{l d}^{*}-i_{l d}\right)+k_{i d} \phi_{d} \\
\frac{d i_{l q}}{d t}= & \frac{d i_{l q}^{*}}{d t}+k_{p q}\left(i_{l q}^{*}-i_{l q}\right)+k_{i q} \phi_{q} \\
\frac{d u_{c}}{d t}= & \frac{3 L_{l}}{2 C u_{c}}\left\{\left[\left(\frac{d i_{l d}^{*}}{d t}+k_{p d}\left(i_{d}^{*}-i_{l d}\right)+k_{i d} \phi_{d}\right.\right.\right. \\
& \left.-\left(-\frac{R_{l}}{L_{l}} i_{l d}+\omega i_{l q}+\frac{v_{l d}}{L_{l}}\right)\right] i_{l d} \\
& +\left[\left(\frac{d i_{l q}^{*}}{d t}+k_{p q}\left(i_{l q}^{*}-i_{l q}\right)+k_{i q} \phi_{q}\right)\right. \\
& \left.\left.-\left(-\frac{R_{l}}{L_{l}} i_{l q}-\omega i_{l d}+\frac{v_{l q}}{L_{l}}\right)\right] i_{l q}\right\}-\frac{i_{c}}{C}
\end{aligned}
$$

It is seen that the surfaces $i_{l d}^{*}-i_{l d}=0$ and $i_{l q}^{*}-i_{l q}=0$ give globally attractive manifolds and the zero dynamics relative to the outputs $y_{2}$ are:

$$
\dot{u}_{c}=-\frac{i_{c}}{C}+\frac{3}{2 C u_{c}}\left[-R_{l}\left(i_{l d}^{*}\right)^{2}+v_{l d} i_{l d}^{*}\right]
$$

and then the linearizd zero dynamics is:

$$
B=\left.\frac{\partial \dot{u}_{c}}{\partial u_{c}}\right|_{u_{c}^{*}}=\frac{3\left[R_{l}\left(i_{l d}^{*}\right)^{2}-v_{l d} i_{l d}^{*}\right]}{2 C\left(u_{c}^{*}\right)^{2}}
$$


A positive $i_{c}$ means that the AC network supplies the power, which equals $v_{l d} i_{l d}^{*}$, to the HVDC grid. In practice, $v_{l d} i_{l d}^{*}$ should be much larger than the losses represented by $R_{l}\left(i_{l d}^{*}\right)^{2}$. Thus, the term $R_{l}\left(i_{l d}^{*}\right)^{2}-v_{l d} i_{l d}^{*}$ results in $B<0$. Therefore, the zero dynamics (37) is well behaved and locally stable. The overall system (34)-(36) is locally asymptotically stable [11] and the converter can operate in rectification mode. Now the crucial step in this procedure is to determine the reference value of $i_{l d}$ which is not directly given but might be deduced from the known $u_{c}^{*}$ and $i_{l q}^{*}$. In this paper, $i_{l d}^{*}$ is developed based on a simplified model.

By considering that when $i_{l d}$ and $i_{l q}$ converge to $i_{l d}^{*}$ and $i_{l q}^{*}$, (3) can be replaced by the following simplified model:

$$
\frac{d u_{c}}{d t}=-\frac{i_{c}}{C}+\frac{3}{4 C}\left(M_{d}^{\prime} i_{l d}^{*}+M_{q}^{\prime} i_{l q}^{*}\right)
$$

where

$$
\left(\begin{array}{c}
M_{d}^{\prime} \\
M_{q}^{\prime}
\end{array}\right)=\frac{2}{u_{c}}\left(\begin{array}{l}
-R_{l} i_{l d}^{*}+\omega L_{l} i_{l q}^{*} \\
-R_{l} i_{l q}^{*}-\omega L_{l} i_{l d}^{*}
\end{array}\right)
$$

and $i_{l d}^{*}$ can be viewed as the control input. We would like to develop a strategy for $i_{l d}^{*}$ such that $u_{c}$ in (39) is stabilized at $u_{c}^{*}$. Since $\left(i_{l d}^{*}\right)^{2}$ explicitly appears in (39), we then add an integrator in $i_{l d}^{*}$ which yields a higher order system as:

$$
\begin{aligned}
\frac{d u_{c}}{d t} & =-\frac{i_{c}}{C}+\frac{3}{2 C} \frac{\left(v_{l d} i_{l d}^{*}-R_{l} i_{l d}^{*^{2}}\right)+\left(v_{l q} i_{l q}^{*}-R_{l} i_{l q}^{*^{2}}\right)}{u_{c}} \\
\frac{d i_{l d}^{*}}{d t} & =u_{d}
\end{aligned}
$$

The above system is in a standard form:

$$
\dot{x}_{0}=f_{0}\left(x_{0}\right)+g_{0} u_{d}
$$

with the trivial expressions for $f_{0}$ and $g_{0} \in \mathbb{R}^{2}$ where $x_{0} \triangleq$ $\left(\begin{array}{ll}u_{c} & i_{l d}^{*}\end{array}\right)^{T}$. We define $u_{c}$ as the output and then the relative degree of $u_{c}$ is 2 . Consequently, the following expressions can be deduced:

$$
\begin{aligned}
& \dot{u}_{c}=L_{f_{0}}^{1}\left(u_{c}\right) \\
& \ddot{u}_{c}=L_{f_{0}}^{2}\left(u_{c}\right)+L_{g_{0}} L_{f_{0}}^{1}\left(u_{c}\right) u_{d}
\end{aligned}
$$

where

$$
\begin{aligned}
& L_{f_{0}}^{1}\left(u_{c}\right)=- \frac{i_{c}}{C}+\frac{3}{2 C} \times \\
& \frac{\left(-R_{l} i_{l d}^{2}+v_{l d} i_{l d}^{*}\right)+\left(-R_{l} i_{l q}^{*^{2}}+v_{l q} i_{l q}^{*}\right)}{u_{c}} \\
& L_{f_{0}}^{2}\left(u_{c}\right)=- \frac{3 \dot{u}_{c}}{2 C} \frac{\left(-R_{l} i_{l d}^{*^{2}}+v_{l d} i_{l d}^{*}-R_{l} i_{l q}^{*^{2}}+v_{l q} i_{l q}^{*}\right)}{u_{c}^{2}} \\
& L_{g_{0}} L_{f_{0}}^{1}\left(u_{c}\right)=\frac{3}{2 C} \frac{1}{u_{c}}\left(-2 R_{l} i_{l d}^{*}+v_{l d}\right)
\end{aligned}
$$

Hence, we can define the change of coordinates as:

$$
\begin{aligned}
& z_{1}=u_{c} \\
& z_{2}=L_{f_{0}}^{1}\left(u_{c}\right)
\end{aligned}
$$

in which the system (39)-(42) is rewritten as:

$$
\begin{aligned}
& \dot{z}_{1}=z_{2} \\
& \dot{z}_{2}=L_{f_{0}}^{2}\left(u_{c}\right)+L_{g_{0}} L_{f_{0}}^{1}\left(u_{c}\right) u_{d}
\end{aligned}
$$

TABLE I

THE VSC TERMINAL PARAMETERS

\begin{tabular}{c|c|c|c|c}
$R_{l}$ & $L_{l}$ & $V_{\text {line }}$ & $C$ & $f$ \\
\hline $10.1 \mathrm{~m} \Omega$ & $3.2 \mathrm{mH}$ & $415 \mathrm{~V}$ & $680 \mu \mathrm{F}$ & $50 \mathrm{~Hz}$
\end{tabular}

TABLE II

BASE QUANTITIES APPLIED IN THE PER-UNIT SYSTEM.

$$
\begin{array}{l||l|l}
\text { AC side } & S_{b}=10 \mathrm{kVA} & V_{b}=415 \sqrt{2} \mathrm{~V} \\
\hline \text { DC side } & S_{d c, b}=10 \mathrm{kVA} & V_{d c, b}=730 \mathrm{~V}
\end{array}
$$

By introducing a synthetic input $\theta_{d}, u_{d}$ is designed as

$$
u_{d}=\frac{1}{L_{g_{0}} L_{f_{0}}^{1}\left(u_{c}\right)}\left(-L_{f_{0}}^{2}\left(u_{c}\right)+\theta_{d}\right)
$$

where $\theta_{d}$ is chosen using linear techniques as:

$$
\theta_{d}=-c_{1}\left(u_{c}-u_{c}^{*}\right)+c_{2} z_{2}-c_{3} \phi_{u}
$$

where $c_{1}, c_{2}$ and $c_{3}$ are positive. Finally, $i_{l d}^{*}$ is developed as:

$$
\frac{d i_{l d}^{*}}{d t}=\frac{-L_{f_{0}}^{2}\left(u_{c}\right)-c_{1}\left(u_{c}-u_{c}^{*}\right)-c_{2} z_{2}+c_{3} \phi_{u}}{L_{g_{0}} L_{f_{0}}^{1}\left(u_{c}\right)}
$$

Remark 1: From the above description, a reference trajectory $i_{l d}^{*}$ is deduced from the simplified model by adding the additional integrator. The dynamics of $i_{l d}^{*}$ can be controlled by regulating the control gains $c_{1}, c_{2}$ and $c_{3}$. Thus, when implementing $v_{d}$ in (31), we usually remove the feedforward term $\frac{d i_{l d}^{*}}{d t}$ which can be regulated to have much slower dynamics compared with $i_{l d}$. This action makes the controller much easier to be implemented and represents a small additive disturbance in a stable system.

Remark 2: In the case of $i_{c}=0$, we can also apply the control strategy (30) under condition that $u_{c}$ in (30) must be replaced by $u_{c}^{*}$ to eliminate the static error.

\section{Simulation Results}

In order to validate the developed control structure, simulations are performed using MATLAB/Simulink. The parameters of the VSC terminal are listed in Table I. The base quantities used in the per-unit system are in Table II.

As previously described, the control system consists of two parts, i.e. the static feedback linearization control law and the dynamic one based on the simplified model. The direction of $i_{c}$ is the key to determine which one is applied to the VSC terminal. In order to evaluate the performances of the control structure, several simulation scenarios are considered in this paper and all of them just use the exact feedback linearization, which means that the integral part was simplified in the control system.

\section{A. Performance evaluation of the static feedback lineariza- tion control law}

In this scenario, the converter operates in inversion mode. At the start of the simulation, the system works in a state with $u_{c}=1.0$ p.u., $i_{c}=-0.219$ p.u., $i_{l q}=0.01$ p.u. and $i_{l d}=-0.219$ p.u.. 
Before $t=6 \mathrm{~s}, i_{c}$ is subjected to a step change every one second, as shown in Fig. 2(a). New reference value of $u_{c}^{*}$ is given $t=8 \mathrm{~s}$ as illustrated in Figs. 2(d).

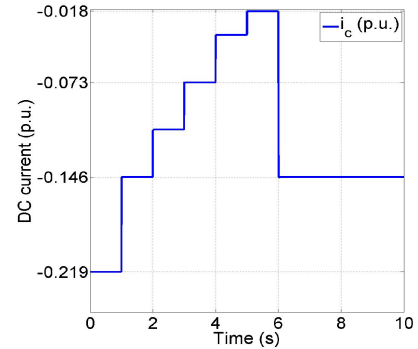

(a) Sequence of events applied to $i_{c}$.

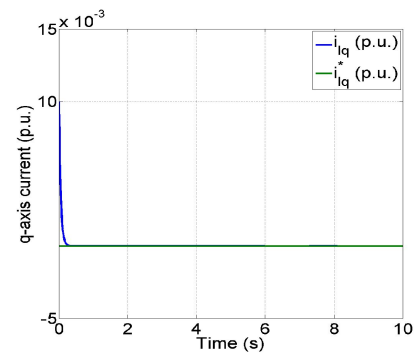

(c) $i_{l q}$ and its reference.

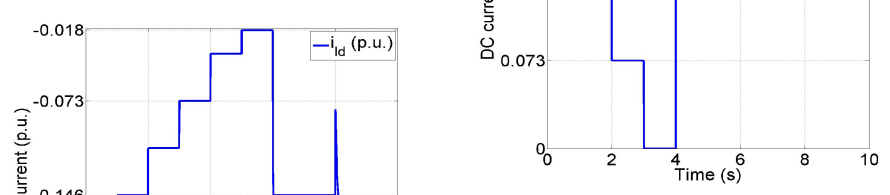

(a) Sequence of events applied to $i_{c}$.

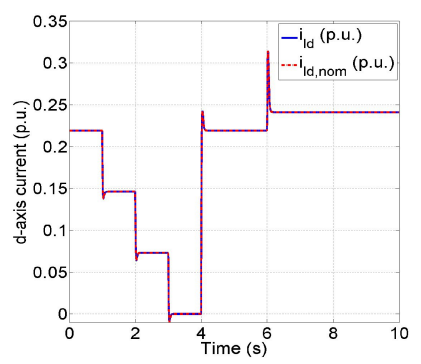

(c) $i_{l d}$ and $i_{l d, \text { nom }}$.

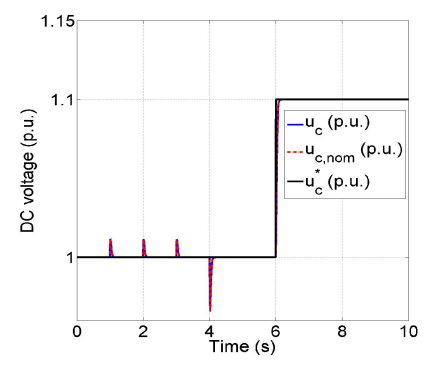

(b) $u_{c}, u_{c, \text { nom }}$ and $u_{c}^{*}$.

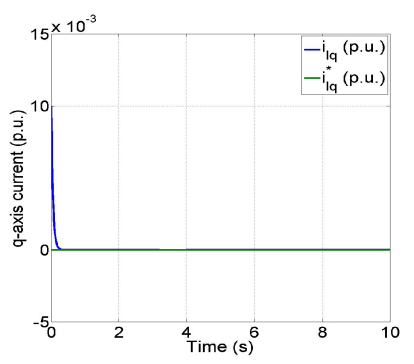

(d) $i_{l q}$ and $i_{l q}^{*}$.
Fig. 3. Simulation results with the feedback linearization control law based on the simplified model.

As presented in Fig. 3(c), $i_{l d}$ reaches the corresponding steady state every time $i_{c}$ varies. We also find that the trajectories of $i_{l d}$ and $i_{l d, \text { nom }}$ almost coincide with each other as seen in Fig. 4(b). Figure 5(b) clearly shows that the discrepancy between $i_{l d}$ and $i_{l d, \text { nom }}$ is extremely small.

The response of DC voltage are shown in Fig. 3(b). If $u_{c}^{*}$ is kept unchanged, $u_{c}$ is well controlled at 1 p.u. in spite of the change of $i_{c}$. At $t=6 \mathrm{~s}, u_{c}^{*}$ is increased by $10 \%$ as in Section IV-A. $u_{c}$ gives a fast response and converges to the new reference value, i.e. 1.1 p.u. As seen in Fig. 4(a), the response of $u_{c}$ is almost identical to that of $u_{c \text {,nom. The error }}$ between the two responses is very small (see Fig. 5(a)).

As shown in Fig. 3(d), although $i_{l q}$ starts from the point $i_{l q}=0.01$ p.u., it quickly converges to its reference value, i.e. $i_{l q}^{*}=0$ p.u..

The above simulation results illustrate that we can use the simplified model (41)-(42) to generate the reference signal for $i_{l d}$ such that $u_{c}$ can be well regulated at its desired value.

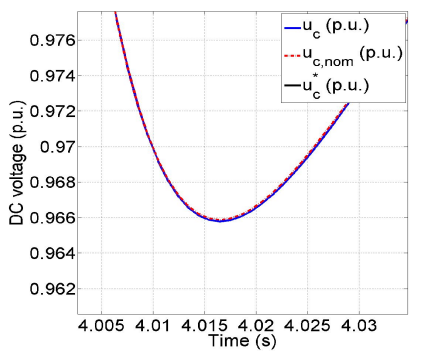

(a) Zoom of $u_{c}$ around $t=4 \mathrm{~s}$.

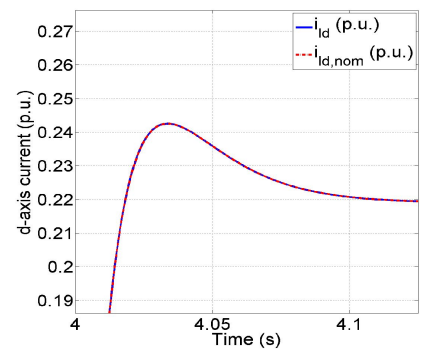

(b) Zoom of $i_{l d}$ around $t=4 \mathrm{~s}$.
0.219 p.u., $i_{l q}=0.01$ p.u. Then, every one second we give a new value to $i_{c}$ until $t=4 \mathrm{~s}$ as shown in Fig. 3(a). In the simulation results, we denote the solutions of the simplified model (41)-(42) as $i_{l d, \text { nom }}$ and $u_{c, \text { nom }}$.
Fig. 4. The responses of $u_{c}$ and $i_{l d}$ around $t=4 \mathrm{~s}$. 


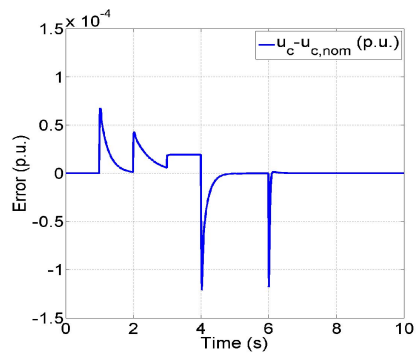

(a) Error between $u_{c}$ and $u_{c \text {,nom. }}$.

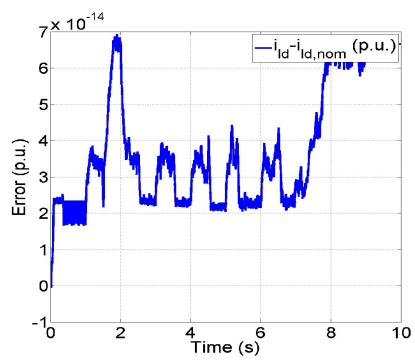

(b) Error between $i_{l d}$ and $i_{l d \text {,nom }}$.

Fig. 5. Error between the physical model and the simplified model.

\section{Performance evaluation of the combination of the static and the dynamic feedback linearization control law}

In this scenario, we evaluate the system performance when the converter operates from one operation mode to the other mode. As depicted in Fig. 6(a), $i_{c}$ reverses from the negative to the positive, which requires the converter to operate from inversion mode to rectification mode.

Before $t=6 \mathrm{~s}$, since $i_{c}$ is negative, the static feedback control law is applied to the VSC terminal. As illustrated in Fig. 6(b) and Fig. 6(d), $u_{c}$ and $i_{l q}$ are well controlled at their respective desired values. During $t \in(6,8) \mathrm{s}, i_{c}=0$. As mentioned in Remark 2, the DC voltage can be kept at its desired level under the controller. When $i_{c}$ reverses, the feedback linearization control law based on the simplified model is used. We see that $u_{c}$ is still stabilized at its reference value after a short transient and the trajectories of $u_{c}$ and $u_{c \text {,nom }}$ are very close to each other. Figure 6(c) shows the response of $i_{l d}$. In order to keep the power balance on both sides of the converter, $i_{l d}$ is brought into a new steady state every time $i_{c}$ or $u_{c}^{*}$ changes.

The simulation results clearly shows that the proposed control structure can make the VSC terminal fulfill bidirectional power transfer.

\section{CONCLUSion}

Focusing on the DC voltage control of a VSC terminal using feedback linearization, this paper shows that the converter is feedback linearizable in inversion mode but not in rectification mode when $u_{c}$ and $i_{l q}$ are defined as the outputs. In order to overcome this limitation, a simplified model is developed to generate a reference signal for $i_{l d}$, since the system is feedback linearizable in rectification mode by defining $i_{l, d q}$ as the outputs.

The advantage of this control system is that we can use linear techniques to design the closed-loop system since the partial nonlinearity of the system can be canceled. As seen from the simulation results, when the converter operates in inversion mode, the performance of the system is good enough since there is almost no overshoot. Therefore, the proposed control system is especially suitable for such VSCHVDC system which is used to transfer the power flow from the unstable energy sources, such as wind farms and solar plants, to the mainland AC network. However, the main drawback is that the control system is complicated which

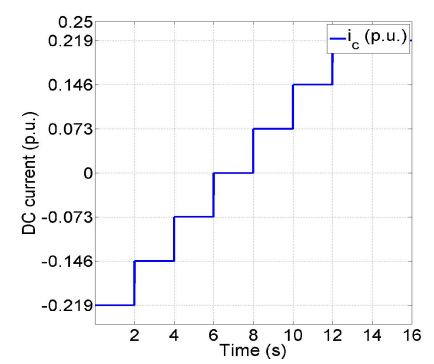

(a) $i_{c}$ varies from negative to positive.

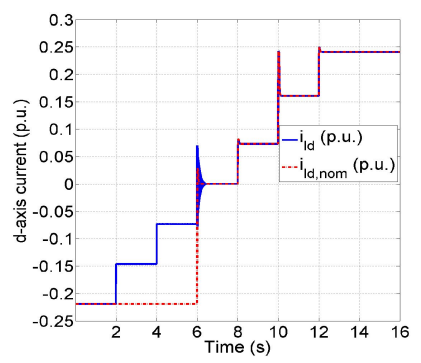

(c) $i_{l d}$ and $i_{l d, \text { nom }}$.

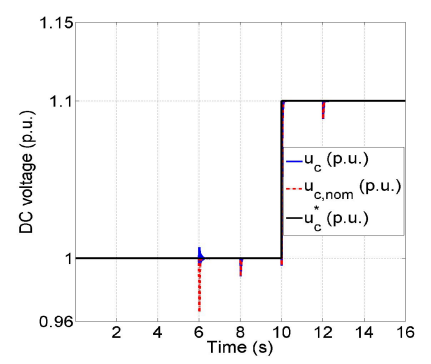

(b) $u_{c}, u_{c, \text { nom }}$ and $u_{c}^{*}$.

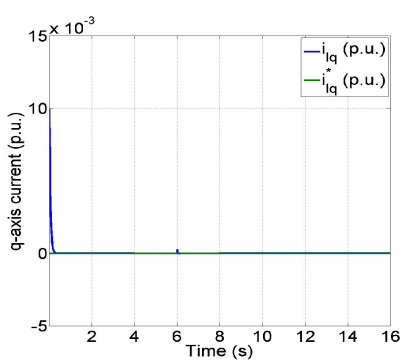

(d) $i_{l q}$ and $i_{l q}^{*}$.
Fig. 6. Simulation results with the proposed control system.

needs to switch between two control laws according to the direction of the power flow, although this case most likely happens only a few times a day.

\section{REFERENCES}

[1] B. T. Ooi, F. Galiana, D. Mcgillis, H. C. Lee, X. Wang, Y. Guo, J. Dixon, H. Nakra, and J. Belanger, "Research in pulse width modulated HVDC transmission," in International Conference on $A C$ and DC Power Transmission, pp. 188-193, September 1991.

[2] L. Xu, B. Williams, and L. Yao, "Multi-terminal DC transmission systems for connecting large offshore wind farms," in IEEE Power and Energy Society General Meeting - Conversion and Delivery of Electrical Energy in the 21st Century, pp. 1-7, July 2008.

[3] J. Liang, T. Jing, O. Gomis-Bellmunt, J. Ekanayake, and N. Jenkins, "Operation and control of multiterminal HVDC transmission for offshore wind farms," IEEE Transactions on Power Delivery, vol. 26, no. 4, pp. 2596-2604, 2011.

[4] A. Reidy and R. Watson, "Comparison of VSC based HVDC and HVAC interconnections to a large offshore wind farm," in Power Engineering Society General Meeting, 2005. IEEE, pp. 1-8, IEEE, 2005.

[5] T. Haileselassie, T. Undeland, and K. Uhlen, "Multiterminal HVDC for offshore wind farms-control strategy," in Wind Power to the GridEPE Wind Energy Conferece, (Stockholm, Sweden), 2009.

[6] A. Lindberg and L. Lindberg, "Inner current loop for large voltage low switching frequency," in 5th International Conference on Power Electronics and Variable-Speed Drives, pp. 217-222, October 1994.

[7] S. Li, T. A. Haskew, and L. Xu, "Control of HVDC light system using conventional and direct current vector control approaches," IEEE Transactions on Power Electronics, vol. 25, no. 12, pp. 3106-3118, 2010.

[8] Y. Chen, A. Benchaib, G. Damm, M. Netto, F. Lamnabhi-Lagarrigue, et al., "Control induced explicit time-scale separation to attain dc voltage stability for a vsc-hvdc terminal," in 19th IFAC World Congress (Accepted), (Cape Town, South Africa), August 2014.

[9] I. Martinez-Perez, G. Espinosa-Perez, G. Sandoval-Rodriguez, and A. Doria-Cerezo, "IDA passivity-based control of single phase backto-back converters," in IEEE International Symposium on Industrial Electronics, pp. 74-79, IEEE, 2008.

[10] J. L. Thomas, S. Poullain, and A. Benchaib, "Analysis of a robust DC-bus voltage control system for a VSC transmission scheme," in 7th International Conference on AC and DC Transmission, 2001.

[11] A. Isidori, Nonlinear Control Systems, Third Edition. Springer, 1995. 